\title{
Turbulent flame shape switching at conditions relevant for gas turbines*
}

\author{
I. Langella $a^{1,3 \dagger} \quad$ J. Heinze ${ }^{2}$ N. Swaminathan ${ }^{3}$, \\ T. Behrendt ${ }^{2}, \quad$ L. Voigt ${ }^{2}, \quad$ M. Zedda ${ }^{4}$ \\ ${ }^{1}$ Department of Aeronautical and Automotive Engineering, \\ Stewart Miller building, Loughborough University, LE11 3TU, UK \\ ${ }^{2}$ Institute of Propulsion Technology, German Aerospace Centre \\ (DLR), Linder Hoehe, Cologne 51147, Germany \\ ${ }^{3}$ Department of Engineering, Trumpington Street, University of \\ Cambridge, CB2 1PZ, UK \\ ${ }^{4}$ Combustion Aerothermal Methods, Rolls-Royce plc, PO Box 31, \\ Derby DE24 8BJ, UK
}

\begin{abstract}
A numerical investigation is conducted in this work to shed light on the reasons leading to different flame configurations in gas turbine combustion chambers of aeronautical interest. Large eddy simulations (LES) with a flamelet-based combustion closure are employed for this purpose to simulate the DLR-AT Big Optical Single Sector (BOSS) rig fitted with a Rolls-Royce developmental lean burn injector. The reacting flow field downstream this injector is sensitive to the intricate turbulent-combustion interaction and exhibits two different configurations: (i) a penetrating central jet leading to an M-shape lifted flame; or (ii) a diverging jet leading to a V-shaped flame. First, the LES results are validated using available BOSS rig measurements, and comparisons show that the numerical approach used is consistent and works well. The turbulent-combustion interaction model terms and parameters are then varied systematically to assess the flame behavior. The influences observed are discussed in the paper from physical and modelling perspectives to develop physical understanding on the flame behavior in practical combustors for both scientific and design purposes.
\end{abstract}

\footnotetext{
${ }^{*}$ The presented manuscript is a copy of the ASME paper GT2019-91879, made by the same authors. ASME is the original publisher of this work, and the sole copyright holder. Any redistribution of this work must fall under the obligations given by this copyright.

${ }^{\dagger}$ Corresponding author, i.langella@lboro.ac.uk
} 


\section{Introduction}

Gas turbine burners are designed to have flames stabilized and located away from metal parts such as injectors and combustor walls to avoid potential damages of the structural components. Among those, lean-burning operating combustors have recently captured the industry interest because of their potential to reduce NOx emissions. The combustion in these devices is unduly turbulent and only partially premixed because of the short resident times typical of modern engines $[1,2]$. As a consequence, the flame propagates through an inhomogeneous mixture, and thus it is prone to oscillations that can affect the surrounding turbulent flow field. Turbulence and coherent flow structures present in the flow can in return interact with the flame, affecting heat release (HR) and flame propagation speed $[3,4,5]$. The HR can further interact with acoustic waves, which can lead to thermo-acoustic instabilities under certain conditions $[6,7]$. When the flow is swirled, additional unsteady phenomena can also occur such as flashback and auto-ignition $[8,9]$, or a precessing vortex core (PVC), which can drive the coupling between acoustics, combustion and swirled flow $[3,4,10]$. Even if stable, the combusting flow may exhibit a periodic motion of velocity and heat release [10,11], which can lead to oscillations of equivalence ratio and generation of rich pockets of mixture that can also affect the flame stability [4, 12]. These combustor complex flow features have been studied in many past works, see above refs for a review, and can potentially lead to flame flash-back which can alter the flame location, shape and its evolution. It is also possible for the flame to change from one stable shape to another $[13,12,10,14]$, which may be undesirable from a practical perspective. Thus, understanding the causes for the changes in flame shapes is beneficial for the development of future injectors and combustors for gas turbine engines. The interplay between incoming turbulence and combustion, and their effect on the flame shape and position is in particular the focus of this work.

Measurements in practical configurations such as high-pressure rigs of aeronautical interest are challenging because of the cost associated with non-intrusive techniques at high pressure and because of safety reasons [15]. This limits the extent of experimental data available in the literature at these conditions, which would be useful for both the investigation of the physics inside practical devices and for model validation purposes. One practical configuration where experimental data is available is the DLR-AT Big Optical Single Sector (BOSS) rig, which was studied experimentally in $[16,17,18]$, and for which spray and $\mathrm{OH}$ concentration statistics have been measured. These statistics can be used for LES validation purposes. In particular, the experimental $\mathrm{OH}$ is shown in this work for the first time. The burner configuration involves a central pilot surrounded by a main jet. Pre-heated air flows through swirlers in the burner, and liquid kerosene is injected through fuel atomisers. This arrangement produces a lifted partially premixed lean flame which stabilises in the shear layers formed along the central and outer recirculation zones ensued from the sudden expansion of the swirling air streams. The central pilot jet in this configuration is sensitive to the surrounding turbulence-flame interaction and in particular it is 
designed to penetrate relatively far downstream into the combustion chamber. For this situation, the flame is mainly attached in the shear layer of central and outer recirculation zones, and has an M-shape. However, the intricate interplay among heat release, mixing and turbulence can lead to opening of the pilot jet, as will be observed in section 5.2, and the formation of a small recirculation region within it, which in turn may lead to a shift from an M-shaped to a $\mathrm{V}$-shaped flame. Thus, the BOSS rig configuration is interesting for both availability of experimental data and to conduct an investigation on these different flame shape configurations.

With the advancement of modern computers, computational fluid dynamics (CFD) is becoming a powerful tool to complement experimental works, in particular where measurements are limited by cost or optical access like in highpressure devices [15]. In particular, large eddy simulation (LES) is increasingly popular as it has potential to capture unsteady flow phenomena at a relatively affordable computational cost compared to other techniques [19]. In a LES, the turbulent scales are resolved down to a cut-off scale $\Delta$, with models to mimic the unresolved, sub-grid scales, and it is commonly accepted that $\Delta$ has to be chosen so that at least $80 \%$ of the turbulent kinetic energy is at supergrid scales in a well-resolved LES [19]. Among the most common sub-grid scale (SGS) models there are thickened flame approach, Linear Eddy model, conditional moment closure, transported PDFs methods and flamelets-based, and a more comprehensive review of these models can be found for example in [20]. In this work a flamelets-based approach with a revised formulation for the sub-grid variance of a reaction progress variable, to be discussed in more details in section 4.1, is employed, which was shown to handle well the delicate turbulence-combustion interaction (TCI) in both fundamental (see for example $[21,22,23]$ ) and practical configurations (see for example [24, 25]), and at a relatively low computational cost compared to other models. Although the use of flamelets-based approaches for gas turbine (GT) combustors is contentious in the classical viewpoints, a number of works (see for example [26, 27, 28, 29]) have shown that flamelet structures are present at the regimes proper of GTs. This is because at these regimes flamelets can be distributed over a wider region yielding a thicker flame brush rather than be thickened by turbulence. In fact, small eddies may not have enough energy to impart significant changes to the flame internal structure [30, 31] and thus the limits of the flamelet assumption are not well defined $[32,20]$. Therefore, a flamelet approach is used for the investigation in this work as it has the advantage of keeping the computational cost affordable on the high-pressure and complex geometry configuration used here, which is relevant from an industrial perspective. Statistics obtained from LES will be compared to available experimental data for validation purposes. It is worth to note that the same analysis could be conducted by employing a different combustion modelling as long as the relevant flow features are captured by the same and a validation analysis is conducted as for this paper.

The objective of this paper is to investigate on the causes leading to one flame configuration or another from both physical and modelling perspectives. This analysis is conducted by systematically varying the TCI parameters in the 

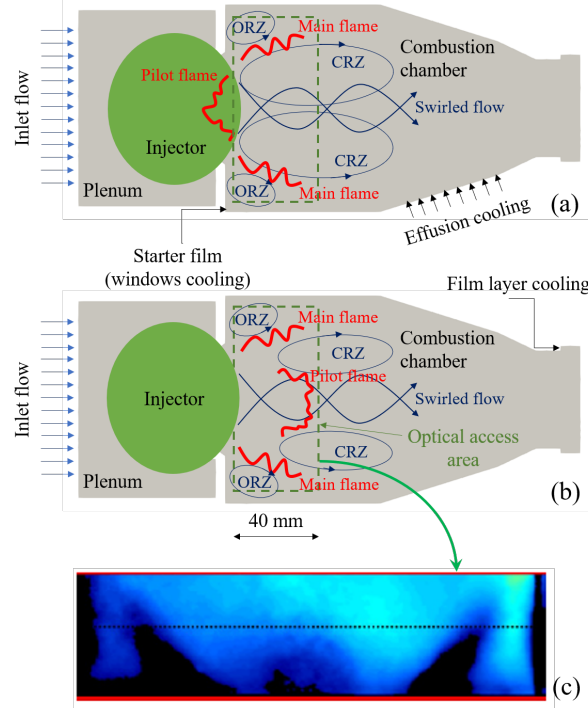

Figure 1: Sketch of the Boss rig geometry showing the V-flame (a) and M-flame (b) configurations. The experimental temperature distribution is shown in (c).

LES model. These variations lead to changes in flame speed and turbulence level, which in turn affect quantities like vorticity, variances and mixing level, eventually determining the flame configuration. The paper is organised as follows: in section 2 the BOSS rig configuration is presented, and the experimental techniques used to collect spray, hydroxyl radicals and temperature statistics are explained in section 3. The numerical details, including combustion model and boundary conditions, are discussed in section 4 . LES results are then compared to experimental data in section 5.1 before the analysis on the flame shapes is conducted in section 5.2. A summary and conclusions are provided in section 6 .

\section{The BOSS rig configuration}

The BOSS rig fitted with a Rolls-Royce developmental lean burn injector is used in this study as test case to conduct the numerical analysis on the different flame configurations discussed in section 1. A sketch of this combustor is shown in Fig. 1, showing the relatively wide optical access in the primary combustion zone and the temperature distribution from experiments to illustrate the flame topology. The particular configuration explored here is typical of take-off conditions and has preheated air at temperature $T_{30}$ above $700 \mathrm{~K}$ and pressure $p_{30}$ above 10 bar entering the squared section of the combustion chamber through a swirler at about $180 \mathrm{~m} / \mathrm{s}$. The injector is based on a two-stage configuration: a pilot jet which flows at the centre of the combustor and a main concentric jet around it. Airblast atomisers are used to spray liquid kerosene (Jet-A1) droplets 
at about $400 \mathrm{~K}$ in both regions, which evaporate and mix with the surrounding air resulting in a air-to-fuel ratio of about 30. Effusion cooling and $1 \mathrm{~mm}$ thick film are used to inject air at ambient and $T_{30}$ temperatures respectively, in order to protect the combustion walls from possible damages due to the high temperatures of the burnt gases. Additional details on this rig can be found in $[16,17,18,33]$.

The flame consists of two branches: the first, main branch stabilises in the shear layer which forms between the central recirculation zone (CRZ) and the outer recirculation zone (ORZ). The second, pilot branch stabilises in the recirculation region created by the swirled flow, where burnt product are entrapped. If the pilot jet is penetrating (as by design conditions), a region of positive axial velocity is present in the region in front of the injector, near the centreline, the pilot flame branch anchors in the inner part of the CRZ and the resulting, overall flame is M-shaped (Fig. 1b). If the pilot jet, on the other hand, enters the combustion chamber with a relatively wide conical angle, the CRZ extends radially inward allowing for negative axial velocities near the centreline. An additional recirculation zone may form in front of the injector and the pilot flame in this condition is a prolongation of the main flame. This results in an overall V-shaped flame (Fig. 1a). These two configurations will be discussed in more details in section 5.2 .

\section{$3 \quad$ Experimental technique and uncertainty}

Measurements in the $40 \mathrm{~mm}$ wide optical access region of the BOSS-rig were taken at DLR and include OH-PLIF to measure $\mathrm{OH}$ concentration, and PDA to characterise the spray in terms of Sauter mean diameter (SMD) and droplet velocity. The experimental setup of the PDA measurements at the BOSS rig and a detailed analysis of the results can be found in [17]. To characterise the flame structure the PLIF of the $\mathrm{OH}$ radical is used as indicator of heat release and temperature. The detailed description on spectroscopic methods and their application in combustor test rigs can be found in [18, 34, 35]. In technical combustion systems like the BOSS rig the PLIF measurement leads generally to the problems of laser absorption and interference of $\mathrm{OH}$ and kerosene LIF. The simultaneous laser absorption and OH-PLIF measurements enable the correction of the PLIF images and the calibration of the OH-PLIF images to absolute OH concentration on single-image basis. The simultaneous detection of the PLIF signal by two cameras using different spectral filters reduces the interference difficulties substantially.

The PDA has an error of respectively $3 \%$ and $10 \%$ respectively in nonreacting and reacting regions. The OH-PLIF technique was reported in [34] to have an error of about $20 \%$ for the $\mathrm{OH}$ concentration distribution, which goes up to $30 \%$ in the proximity of liquid or gaseous kerosene mainly because of the uncertainty of the $\mathrm{OH}$ and kerosene fluorescence quantum. Temperature can be estimated from the strongly temperature dependent $\mathrm{OH}$ concentration using equilibrium assumption and the method explained in [34]. It was shown that in 
this case the error on the measured $\mathrm{OH}$ concentration introduces a corresponding error in the temperature of $40 \mathrm{~K}$ to $65 \mathrm{~K}$ for lean flame configurations such as the one investigated here. The assumption of chemical equilibrium introduces an additional overestimation of temperature within the flame front due to the presence of $\mathrm{OH}$ super-equilibrium concentrations. The analysis in [34] for a flame at 10 bar pressure showed that the reacting mixture relaxes to its equilibrium condition within $50 \mu \mathrm{s}$ and this was shown to correspond to an error on the $\mathrm{OH}$-estimated temperature of about $100 \mathrm{~K}$ within this time.

\section{LES details}

\subsection{Combusiton model}

The partially premixed combustion in the LES model used for this work is handled using a database of premixed flamelets. It is assumed that the smallest turbulent eddies are not small enough to penetrate, or do not last long enough to impart significant changes to the flame reaction zone $[30,20]$ (flamelet assumption). Thus, the turbulent flame is seen as an ensemble of thin, onedimensional laminar flames (flamelets) and the thermochemistry is computed a priori. Although the flamelet assumption is arguable at conditions for aeroengines, past studies have shown that flamelets-like structures are still observed in such regimes (see for example $[31,36,26,37,38,22]$ ).

Two control variables, one for the mixing and one for the rate of reaction progress, are needed to access the database of premixed flamelets. The mixing is tracked using the Bilger's mixture fraction [39], $\xi$ and its Favre-filtered transport equation is

$$
\bar{\rho} \frac{D \widetilde{\xi}}{D t}=\nabla \cdot\left[\left(\bar{\rho} \widetilde{\mathcal{D}}+\frac{\mu_{t}}{S c_{t}}\right) \nabla \widetilde{\xi}\right]+\overline{\dot{\omega}}_{S}
$$

In the above equation $S c_{t} \approx 0.7$ is the SGS Schmidt number and $\widetilde{\mathcal{D}} \approx \widetilde{\mu} / 0.7$ is the filtered molecular diffusivity, which is assumed to be the same for all scalars. The filtered kinematic viscosity, $\widetilde{\mu}$, is computed via Sutherland's law. The SGS viscosity is denoted as $\mu_{t}$. A source term, $\overline{\dot{\omega}}_{S}$, is included in the equation to account for the evaporation of fuel droplets, and it is modelled as in [40, 33]. The reaction progress is tracked using a progress variable, defined in this work as $c=Y_{\mathrm{CO}_{2}} / W_{\mathrm{CO}_{2}}+Y_{\mathrm{CO}} / W_{\mathrm{CO}}$, where $Y_{i}$ and $W_{i}$ are respectively mass fraction and molecular weight of species $i$. This definition is chosen based on previous work [22], and as it guarantees monotonicity on the rich side of combustion [41]. The Favre-filtered transport equation for $c$ is:

$$
\bar{\rho} \frac{D \widetilde{c}}{D t}=\nabla \cdot\left[\left(\overline{\rho \mathcal{D}}+\frac{\mu_{t}}{S c_{t}}\right) \nabla \widetilde{c}\right]+\overline{\dot{\omega}}_{c}
$$

where $\overline{\dot{\omega}}_{c}$ is the filtered reaction rate computed as

$$
\overline{\dot{\omega}}_{c}=\int_{0}^{1} \int_{0}^{1} \dot{\omega}(\zeta, \eta) P(\zeta, \eta) \mathrm{d} \zeta \mathrm{d} \eta,
$$


In the above equation $\zeta$ and $\eta$ are sample space variables for $\hat{c}$ and $\xi$ respectively, where $\hat{c}$ is $c$ normalised with its maximum value for each flamelet so that it varies between zero and one before the integration is performed. The flamelet reaction rate $\dot{\omega}(\zeta, \eta)$ is obtained from unstrained planar laminar premixed flames computed over the whole range of $\xi$ spanning the flammability limits. The SGS joint PDF in Eq. (3) is expressed as $P(\zeta, \eta)=P(\zeta) P(\eta \mid \zeta)$ using Bayes' theorem, where the PDFs of $c$ and $\xi$ are modelled using Beta functions [42]. These Beta functions require respectively the SGS variances of $\xi$ and $c$, defined as $\sigma_{\phi}^{2}=\widetilde{\phi^{2}}-\widetilde{\phi}^{2}$, where $\phi$ is either the mixture fraction or the progress variable. These are computed in the LES from their transport equations:

$$
\begin{array}{r}
\bar{\rho} \frac{D \sigma_{\xi, \mathrm{sgs}}^{2}}{D t}=\nabla \cdot\left[\left(\overline{\rho \mathcal{D}}+\frac{\mu_{t}}{S c_{t}}\right) \nabla \sigma_{\xi, \mathrm{sgs}}^{2}\right] \\
-2 \bar{\rho} \widetilde{\varepsilon}_{\xi}+2 \bar{\rho} \frac{\nu_{t}}{S c_{t}}(\nabla \tilde{\xi} \cdot \nabla \tilde{\xi})+\bar{\rho}\left(2 S^{+}-S^{-}\right),
\end{array}
$$

where the last two terms, $S^{+}$and $S^{-}$, represent production and sink contributions of the droplet evaporation to the SGS variance [43], and

$$
\begin{aligned}
\bar{\rho} \frac{D \sigma_{c, \mathrm{sgs}}^{2}}{D t} \approx & \nabla \cdot\left[\left(\overline{\rho \mathcal{D}}+\frac{\mu_{t}}{S c_{t}}\right) \nabla \sigma_{c, \mathrm{sgs}}^{2}\right]-2 \bar{\rho} \widetilde{\varepsilon}_{c} \\
& +2 \bar{\rho} \frac{\nu_{t}}{S c_{t}}(\nabla \widetilde{c} \cdot \nabla \widetilde{c})+2(\overline{c \dot{\omega}}-\widetilde{c} \overline{\dot{\omega}}) .
\end{aligned}
$$

The reaction source, $\overline{c \dot{\omega}}$, in the above equation is modelled consistently with Eq. (3). A linear relaxation model, $\widetilde{\varepsilon}_{\xi}=C_{\xi}\left(\nu_{t} / \Delta^{2}\right) \sigma_{\xi, \text { sgs }}^{2}$, is used for the SGS scalar dissipation rate (SDR) in Eq. (4), where $\nu_{t}=\mu_{t} / \bar{\rho}$ and $C_{\xi}=2$ [44]. The terms $S^{+}$and $S^{-}$in Eq. (4) where shown to be important under certain conditions, see for example [45], but they were observed to be less important in other works $[43,46]$. These terms have different possible closures and need to be balanced by the SDR of $\xi$, which thus also requires further modelling. Due to this complexity and the contrasting opinions, in this work the interplay between $S^{+}, S^{-}$and the corresponding SDR is assumed to be in perfect balance for simplicity (see for example [46]), while different modelling will be explored in a future study.

An algebraic expression used in many past works [47, 23, 22, 21, 24, 25] is used to model the subgrid SDR of $\widetilde{c}$ in Eq. $5, \widetilde{\varepsilon}_{c}$, which is

$$
\widetilde{\varepsilon}_{c}=\left[1-\exp \left(\frac{-0.75 \Delta}{\delta_{t h}}\right)\right]\left[\left(2 K_{c}-\tau C_{4}\right) \frac{s_{L}}{\delta_{t h}}+C_{3}^{\prime} \frac{\epsilon_{k}}{k}\right] \frac{\sigma_{c, \mathrm{sgs}}^{2}}{\beta_{c}}
$$

In the above expression $\Delta$ is the LES filter size, taken to be the cubic root of the numerical cell volume, and $k=1.5 u_{\Delta}^{\prime 2}$ and $\epsilon_{k}$ are the subgrid kinetic energy and its dissipation rate respectively. The subgrid velocity $u_{\Delta}^{\prime}$ is modelled as [19] $u_{\Delta}^{\prime}=\sum\left(\widetilde{u}_{i}-\widetilde{\widetilde{u}}_{i}\right)$ following previous works [25, 48], where $\widetilde{u}_{i}$ and $\widetilde{\widetilde{u}}_{i}$ are respectively the filtered velocity component in direction $i$ and its test-filtered value obtained using a box filter. It is worth noting that the subgrid kinetic 
energy modelling has to include the flame dilatation effect as shown in [49]. The thermo-chemical parameter $K_{c}$ is $0.79 \tau$, where $\tau=\left(T_{a d}-T_{u}\right) / T_{u}$ is the heat release parameter, and $T_{u}$ and $T_{a d}$ are the reactant and adiabatic flame temperatures respectively. Laminar flame speed, $s_{L}$, thickness, $\delta_{t h}$, and heat release parameter, $\tau$, are taken from the flamelets database. Note that outside the flammability limits $\widetilde{\varepsilon}_{c} \rightarrow \alpha\left(\epsilon_{k} / k\right)\left(\sigma_{c, \text { sgs }}^{2} / \beta_{c}\right)$, where $\alpha$ is a constant. The only adjustable parameter in Eq. (6) is $\beta_{c}$, which is computed dynamically [50, $23,22,21,25]$. All other parameters are non-tunable and signify the combined effect of turbulence and combustion (see more details in [47, 23, 22, 21, 24, 25] ) .

\section{$4.2 \quad$ Numerical setup}

Favre-filtered mass, momentum and energy (total enthalpy) transport equations are solved along with the four equations described in section 4.1. The finite volume approach implemented in the unstructured, low-Mach Rolls-Royce code Precise-UNS [51] is used. A second order implicit Eulerian scheme and the SIMPLEC algorithm [52] with 5 sub-iterations are used for time advancement, while space derivatives are approximated using second order central difference schemes and Gamma limiters in regions of strong gradients. A constant Smagorinsky model is used to close the SGS stresses in the momentum equation, and the gradient hypothesis is used for the turbulent transport term of the scalars. Temperature is estimated from total enthalpy as $\widetilde{T}=\widetilde{T}_{f}+\left(\widetilde{h}-\widetilde{h}_{0}\right) / \widetilde{C}_{p}$, where $\widetilde{T}_{f}, \widetilde{h}_{0}$ and $\widetilde{C}_{p}$ are respectively flamelet temperature, mixture enthalpy at temperature $\widetilde{T}_{f}$ and filtered heat capacity at constant pressure. The second term on the RHS in the previous equation is a correction term to account for subadiabaticity and mixing of streams at different enthalpies, that make the local temperature different from the one in the flamelet database. The mixture density is computed using state equation. The thermodynamic quantities needed to compute $\widetilde{T}$ are obtained using equations similar to Eq. (3), and then tabulated in terms of the four controlling variables, $\widetilde{c}, \widetilde{\xi}, \sigma_{c, \mathrm{sgs}}^{2}$ and $\sigma_{\xi, \mathrm{sgs}}^{2}$. Finally, the look-up table is computed for all flamelets spanning the flammability limits using the in-house Rolls-Royce code Chem1d and the Dagaut mechanism [53] for the kerosene-air thermo-chemistry. A modified version of the Rolls-Royce in-house PPDF code is used for the presumed-PDF integration. The resulting look-up table has respectively 57, 100 and 50 linearly distributed point respectively for Favre-filtered mixture fraction, scaled progress variable and its SGS variance. The mixture fraction SGS variance has instead 15 non-uniformly distributed points between zero and $\widetilde{\xi}(1-\widetilde{\xi})$, clustering close to the zero. Outside the flammability limits the reaction terms are assigned to zero, while the other thermodynamic quantities are interpolated between the lean (rich) limit and the air (fuel) condition. 


\subsection{Boundary conditions}

The grid used for the BOSS-rig configuration is hexa-dominant with about 14 million cells and a resolution of $\Delta \approx 0.8 \mathrm{~mm}$ in the flame region. This grid includes the plenum upstream and was shown to satisfy the Pope's criterion for turbulent kinetic energy in [33]. The same criterion has been used in this work for the particular operating point investigated here, and shows that the SGS kinetic energy (estimated using the model of Lilly [54]) amounts to less than $10 \%$ of the total (SGS plus resolved) turbulent kinetic energy almost everywhere in the combustor and except for the region close to the injector walls. This is acceptable as the most of the turbulence is produced by the swirl and shear layers for the highly turbulent configuration investigated here. It is worth noting that Pope's criterion is thought for isothermal flows and thus it has to be used with care for reacting flows where local overshoots due to the flame dilatation occur [49].

Boundary conditions are assigned as follows. Velocity is specified according to the mass flow rate at the plenum inlet. Synthetic turbulence is not specified as the turbulence level is small compared to that generated inside the combustion chamber due to swirl and shear layers. Film and effusion cooling boundary surfaces are included in the domain, but due to their relatively small sizes they are treated as walls, where point sources of mass and momentum are homogeneously distributed to match the actual flow rates. Boundary layers near walls are not resolved due to turbulence being mainly produced by shear layers and swirl, and to avoid the significant increase of mesh size in the injector. The velocity is assigned to zero on the walls and a zero gradient condition is given at the outlet. Pressure is assigned to the operating value $p_{30}$ at the outlet, is extrapolated at the inlet and has zero normal gradient on the walls. All other scalars have zero gradients on walls and zero at the inlets, except for enthalpy which is assigned at the inlets according to the corresponding temperature.

A constant time step of $1 \mu \mathrm{s}$ is used for time advancement. This time step guarantees a maximum CFL number of 0.3 in the measurement region. The simulation took about 14 days on a wall clock to simulate about $30 \mathrm{~ms}$ of physical time using 256 cores of Darwin cluster at Cambridge University. These correspond to about 18 flow-through times, where a flow-through time is defined as the time spent by a parcel of fluid to travel the combustion chamber at the bulk velocity just downstream the injector. Of these, about 6 flow-through times where used to allow the transient to pass and additional 12 to collect statistics.

\section{Spray modelling}

A Lagrangian approach is used. Numerical parcels are injected from the edges of the injectors, and sampled using a Rosin-Rammler distribution and the Sattelmayer correlation for the initial SMD [55], which is set in the range of $15-20 \mu \mathrm{m}$, with a dispersion parameter $q$ of about 3.0. The parcels are injected at a constant velocity of about $12 \mathrm{~m} / \mathrm{s}$ and with random injection angle following a Gaussian distribution [45] with mass flow rate given according to experimental 

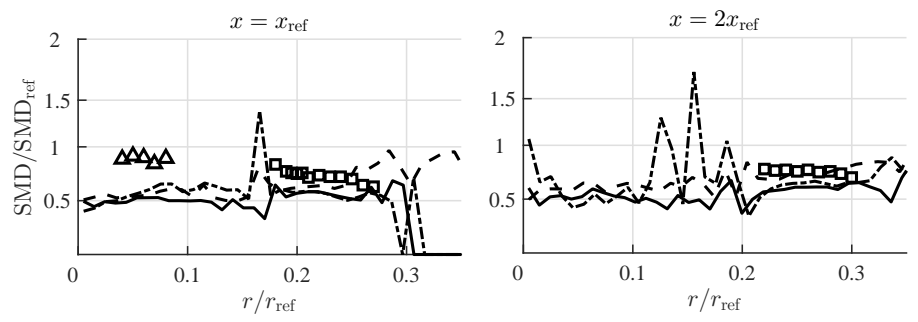

Figure 2: SMD radial variation at different axial positions from measurements (symbols) and LES: cases A (-), C (-·) and S (- -) of Table 1.

data, mean aligned to the injector axis and $10^{\circ} \mathrm{rms}$. Only secondary breakup is considered using the approach described in [56]. A rapid mixing formulation is used to model the droplet evaporation, based on the Spalding mass transfer number with no correction for Stefan flows. The liquid kerosene is assumed to have very large thermal conductivity (very low Biot number), with uniform temperature for the droplet and an evaporation temperature set to $660 \mathrm{~K}$. An equilibrium assumption is used for the computation of the fuel mass fraction at the droplet surface. Further details can be found in [57].

It is worth to note that, in general, spray droplets can enter the flame and affect its structure during the evaporation process. However, for the particular lean direct injection burner investigated here, the most of the fuel has evaporated before entering the flame region. This is supported by experimental observations of comparisons between Mie scattering, Kerosene LIF and $\mathrm{OH}^{*}$ chemiluminescence (not reported here), and partly by the experimental data to be discussed for Fig. 2 in the next section. Thus, although the modelling used presents limitations, it is satisfactory for the purposes of the current analysis and is chosen here for the seek of simplicity.

\section{Results}

\section{$5.1 \quad$ Validation}

The spray droplet breakup and evaporation will determine the local level of mixing, and thus the local heat release rate in the flame area. It is thus important that the droplet velocity and distribution is well captured in the LES. The spray Sauter mean diameter (SMD) and droplet velocity obtained experimentally in [17] for the same reacting case under investigation, are used here to validate the spray model used in this work. Comparisons of measured and computed SMD are shown in Fig. 2 (Case A). The SMD predicted by LES matches fairly well that from experiments in the main region $\left(r / r_{\text {ref }}>0.15\right)$ at all axial positions. In the pilot region the SMD is slightly under-predicted by LES at $x=x_{\text {ref }}$ suggesting a faster breakup or evaporation with respect to 

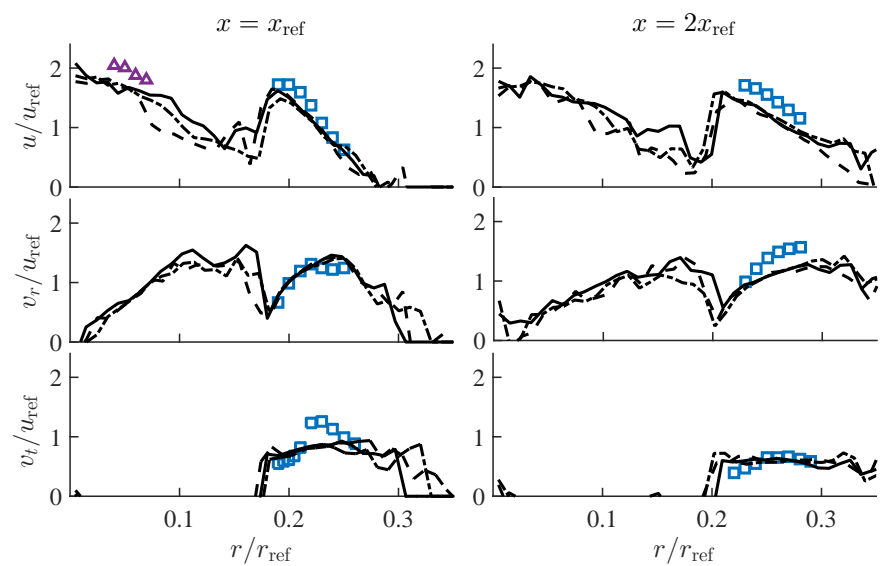

Figure 3: Radial variation of axial, radial and tangential spray velocities at different axial positions from measurements and LES. Legend is as for Fig. 2.

those observed in the experiment. However, further downstream the spray gets very thin and the PDA measurements were limited to regions with a reasonable balance between statistical validity and required measuring time. In the LES the SMD distribution is calculated for all radial positions. Spray velocities from LES, compared to those from experiments in Fig. 3, are also accurate at all axial positions with some under-prediction observed only at the downstream positions for axial and radial velocity. This suggests that the spray model used in this work is accurate.

An additional analysis was conducted to understand the sensitivity of the spray statistics to the combustion model. This was obtained by artificially changing the temperature of about $30 \%$ by varying the model constants in the progress variable variance equation. The simulations used for this analysis are summarised in Table 1. A decrease of temperature field (increase of SGS variance, Case $S$ ) results in an M-flame configuration with increased penetration of the pilot jet into the chamber as one would expect. However, this change only slightly affects the main region but not the pilot region as observed for both SMD and spray velocity statistics. In particular, an increase of SMD, radial and tangential velocities, not observed in the measurements, is observed in the LES at $x / x_{\text {ref }}$ for $r / r_{\text {ref }}>0.28$. When the temperature increases (decrease of SGS variance, Case C) the flame is observed to transition to a V-shape configuration. Still, no significant effect in the spray statistics is observed. This is partly because of the fast evaporation in the pilot region as discussed earlier and partly because the secondary breakup model for the spray may reduce the sensitivity to the flow conditions. This analysis shows that both SMD and droplet velocity are not significantly affected by temperature changes and thus by the combustion model, even if the flame changes location. This information is important for the analysis on flame shapes to be conducted in section 5.2, 
Table 1: Simulations used for sensitivity analysis on the spray statistics.

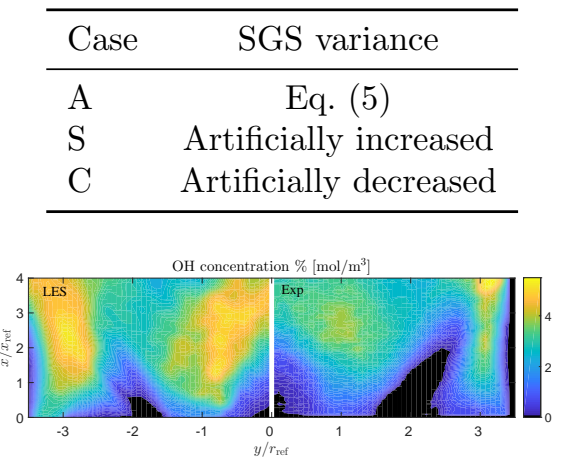

Figure 4: Numerical and experimental mid-plane $\mathrm{OH}$ concentration contours are shown in the optical region of the Boss rig.

where the TCI parameters will also be changed.

To compare the numerical and experimental results of the flame structure, the $\mathrm{OH}$ fields are used instead of the temperature fields for two reasons. On the one hand, the $\mathrm{OH}$ concentration is the measured quantity. Temperature values has to be estimated in the experiments using equilibrium assumption as explained in section 3. This assumption introduces additional uncertainty which would require specific considerations before meaningful comparisons between LES and experimental data can be made. On the other hand, the exponential temperature dependence on the $\mathrm{OH}$ concentration enables temperature evaluation only above $1400 \mathrm{~K}$. Under the present experimental conditions of incipient combustion, a large part of the considered area used for the measurements has less than $50 \%$ of the events that could be used to estimate temperature. Thus, it is preferred to directly compare the numerical and experimental $\mathrm{OH}$ fields, while comparisons of temperature are postponed to a future work. This comparison is shown in Fig. 4. It is worth mentioning that the experimental $\mathrm{OH}$ as shown would not be symmetric around the centreline partly because of the UV laser absorption by kerosene, and partly because of possible geometrical asymmetries that were observed during the experiment in [34]. The numerical $\mathrm{OH}$ concentration is obtained from its mass fraction in the flamelet table as $[\mathrm{OH}]=\rho Y_{\mathrm{OH}} / W_{\mathrm{OH}}$, where $\rho$ is the density from the LES and $W_{\mathrm{OH}}$ is the $\mathrm{OH}$ molar mass. The figure shows that the $\mathrm{OH}$ level is overestimated in the LES as compared to the experiment by about $30 \%$ in the main and pilot reacting regions, where the peak concentrations are observed. Although overestimations of $\mathrm{OH}$ in the flamelets context are somehow expected based on previous studies (see for example [22]), these differences are of the order of the measurement uncertainty reported in section 3 (20\% to $30 \%)$. Moreover, the favourable $\mathrm{OH}-$ temperature relation would imply that differences in temperature field would be smaller than those observed for the $\mathrm{OH}$, although the comparison in Fig. 4 sug- 
gests that the reactions in the main jet region happen slightly more downstream in the experiment than as predicted by the LES. The comparison also suggests that reactants in the main jet are more penetrating in the experiment, although this may be due to the difficulty of measuring $\mathrm{OH}$ concentrations below 1400 K. Overall, the LES framework used here seems to predict the correct flame configuration, which is of great importance for the analysis to be conducted in the next section, and with relatively good accuracy.

\subsection{Flame and flow behaviour}

\subsubsection{Time evolution}

Main and pilot jets are swirled by the injector, and this results in the formation of an central recirculation zone where burnt gases are entrapped, providing the mechanism for the flame to stabilise and self-sustain itself. The jet velocity can form an angle with the mainstream flow direction, which mainly depends on the injector geometry at the nozzle exits, but also on the surrounding reacting flow developing as the swirled jets move into the combustor. For the BOSS rig configuration, this jet angle is observed to evolve in time. The conical angle formed by the pilot jet moves from zero degrees to about $45^{\circ}$ and then back to zero periodically at a frequency of about $400 \mathrm{~Hz}$. This time evolution is useful to understand the mechanism which leads this jet flipping and is thus discussed next.

Figure 5 shows a time sequence of mid-plane contours of velocity and vorticity magnitude, mixture fraction and heat release rate. The latter is used to indicate the position of the flame. Isolines of zero velocity, progress variable (two isolines representing $10 \%$ and $90 \%$ reaction progress) and mixture fraction

for a lean, $\widetilde{\xi}=0.032$ and the stoichiometric, $\widetilde{\xi}=0.063$, values are shown on top of the first three columns respectively to better indicate recirculation zones and flame region. Six times are shown from a random time $t_{0}$ where the pilot jet angle is zero and for increasing times of $\Delta t=0.5 \mathrm{~ms}$. The velocity contour indicates that the pilot jet angle reaches its maximum at time $t_{0}+3 \Delta t$. At this time a small vortex forms within the jet, which is indicated by the near-zero velocity at this location. The shear layer and the consequent region of relatively small velocities forming around this vortex may allow the flame to move upstream and stabilise at this location. By looking at the heat release contours, the pilot flame moves slightly upstream from time $t_{0}+2 \Delta t$ to $t_{0}+3 \Delta t$ and becomes stronger, to then reach its maximum strength $0.5 \mathrm{~ms}$ later, at $t_{0}+4 \Delta t$. However, at this time the small vortex has almost totally disappeared and the flame starts to reduce its strength again and moves slightly downstream. The small vortex forming at $t_{0}+3 \Delta t$ does not last enough to be seen in the mean field, and in fact the mean pilot jet is penetrating with all positive velocities as could be inferred in Fig. 4. This is because when the jet opens stably, as will be also shown later, the recirculation zone forming within it brings hot gases and thus high temperatures would be seen along the centreline just downstream the injector, in Fig. 4. However, a wider jet angle or a longer-lasting vortex could 


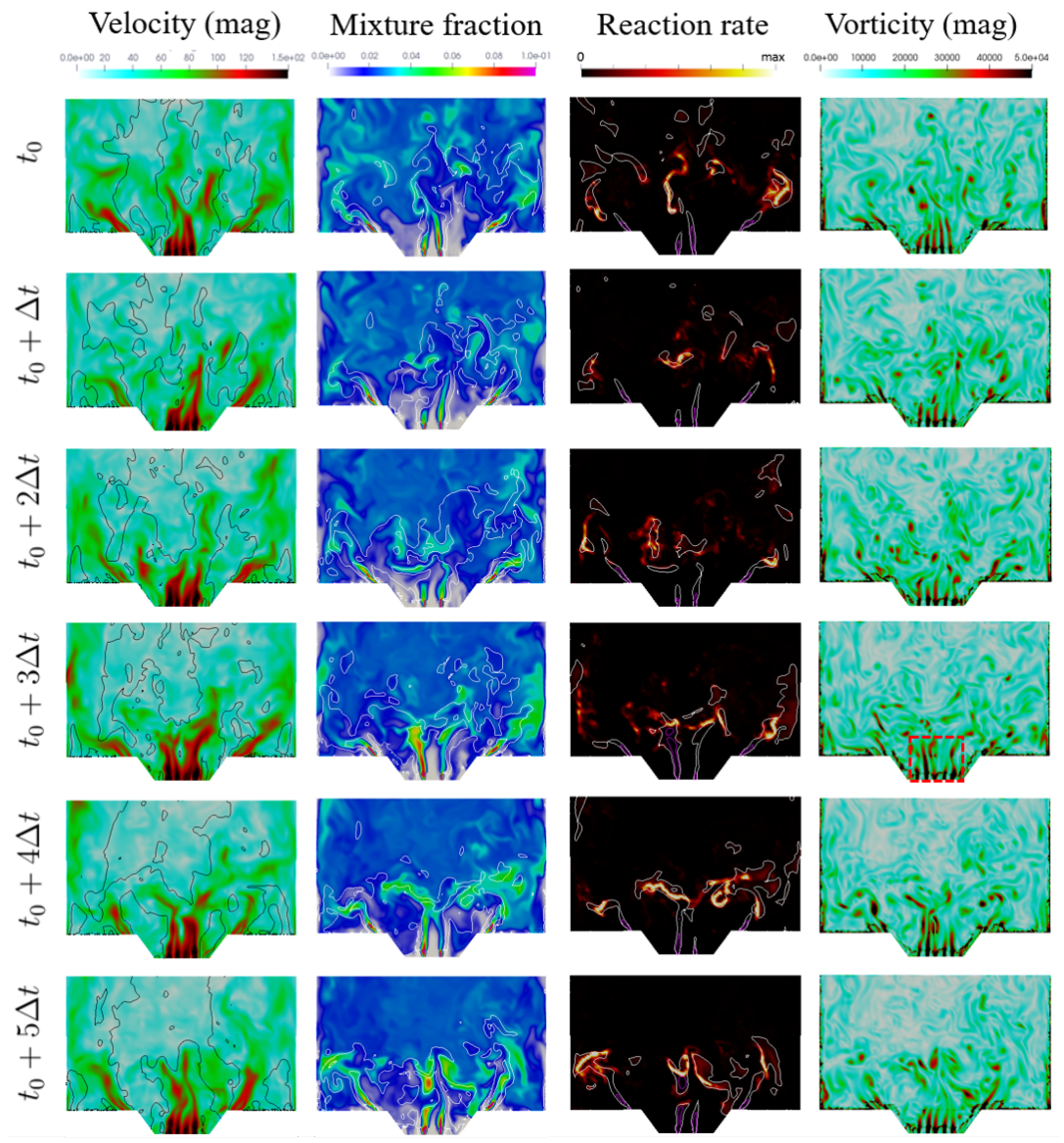

Figure 5: Mid-plane contours of instantaneous velocity (in $\mathrm{m} / \mathrm{s}$ ) magnitude, mixture fraction, heat release rate and vorticity magnitude $\left(\right.$ in $\mathrm{s}^{-1}$ ) are shown in a sequence of six times from $t_{0}$ to $t_{0}+5 \Delta t$, where $\Delta t=0.5 \mathrm{~ms}$. Iso-lines of zero axial velocity (black lines), progress variable (10\% and $90 \%$ reaction progress), and lean $(\widetilde{\xi}=0.032)$ and stoichiometric $(\widetilde{\xi}=0.063)$ mixture fraction (white lines) are shown on top of velocity, mixture fraction and reaction rate contours respectively. 


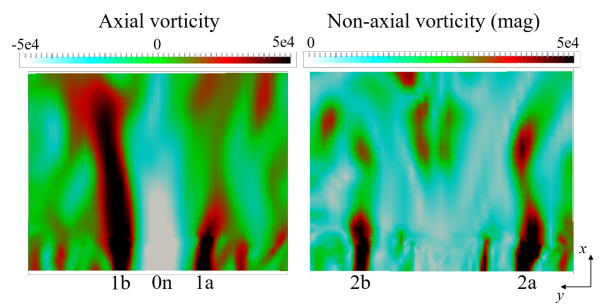

Figure 6: Contours of axial and non-axial (magnitude of the non-axial components) vorticity (in s ${ }^{-1}$ ) at time $t_{0}+3 \Delta t$ in the zoomed region marked in Fig. 5. Five different vortical structures are labelled.

potentially allow the flame to get too close, causing a switch of configuration. It is thus worth to understand the mechanism ruling this.

By looking at the mixture fraction contours in Fig. 5, one can notice that the pilot flame sits in regions of relatively rich mixture as compared to the surrounding region. These patches are also marked by the $\widetilde{\xi}=0.032$ isoline, although within these patches the mixture is always below stochiometric conditions. These relatively rich patches drive the position of the flame upstream from $t_{0}+2 \Delta_{t}$ to $t_{0}+4 \Delta t$. During this evolution the flame is increasing in strength, indicating that it is moving from leaner to richer conditions. This is also demonstrated by the fact that the reaction progress SGS variance (not shown), which could also be responsible for a change in magnitude of the heat release rate, do not show any significant change during the same movement. By a close look at the vorticity field, one can notice that during the evolution towards the maximum jet angle the vorticity brought into the chamber by the pilot jet becomes less uniform and somehow penetrates less near the centreline, and to a minimum extent when the small vortex forms at $t_{0}+3 \Delta t$, to then start to increase again (compare the length of the jet from the velocity contours, which remains about the same, to that from the vorticity contours). Lower vorticity may indicate lower mixing rate, allowing the formation of richer pockets of mixture that produce an increase in flame strength as observed earlier. The conservation of angular momentum implies an energy transfer from axial to azimuthal vorticity as the jet expands under the effect of the radial pressure gradient [58]; thus different vorticity levels can affect this process resulting in different jet expansions. Different vorticity levels can thus imply a flow that may be more prone to pressure gradient effects, and eventually fall apart allowing a vortex to form inside it. This vorticity interchange is studied further here. From Fig. 5 there seem to be five vorticity 'fingers' entering the combustion chamber. Of these, the central one is prevalently produced by negative axial vorticity, probably generated by the swirl. This can be observed from Fig. 6, showing axial and non-axial (magnitude of the two non-axial components) vorticity in the zoomed region indicated for $t=t_{0}+3 \Delta t$ in Fig. 5. By comparison of this vorticity with the total vorticity magnitude of Fig. 5 one can notice that 

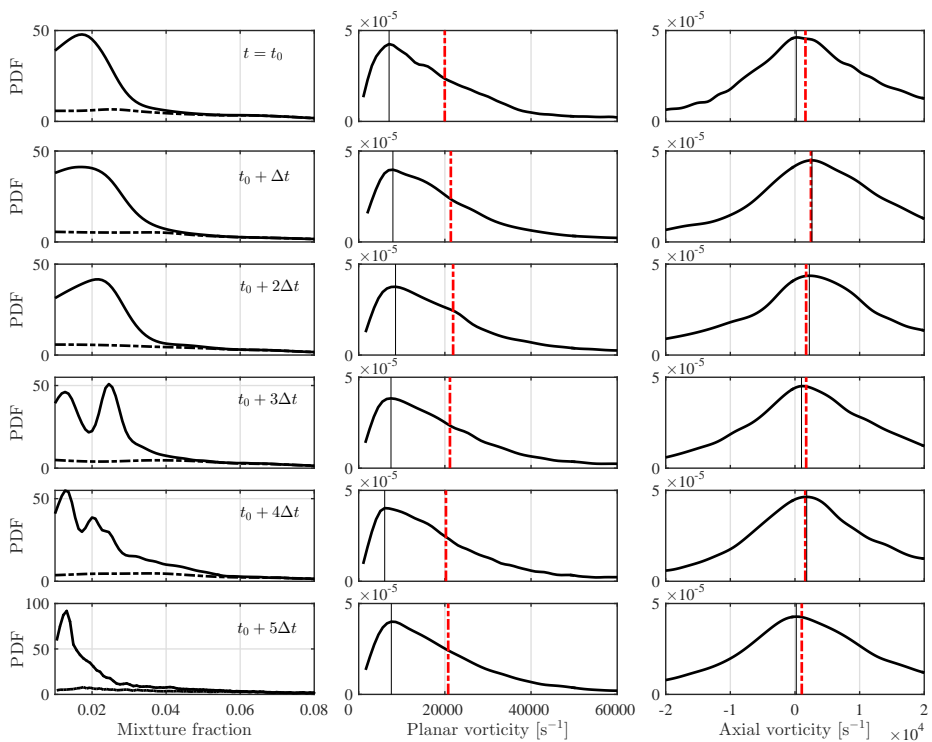

Figure 7: Normalised histograms axial and non-axial (magnitude of the two non-axial components) vorticity in the pilot region for six time steps. Vertical lines indicate peak (-) and mean (- - ) vorticity. Histograms of mixture fraction are also shown in the same region using all (-) and only the samples for $\widetilde{c}<0.1(-\cdot-)$.

the internal 'fingers'( $1 \mathrm{a}$ and $1 \mathrm{~b}$ in the figure) just next to the central one are dominated by positive axial vorticity, while the most external ones (2a and $2 \mathrm{~b}$ in the figure) are dominated by non-axial vorticity, thus vorticity not resulting from the swirl motion. The same qualitative situation occurs also for the other time steps. A more quantitative analysis is given in Fig. 7, where normalised histograms of axial and planar (non axial) vorticity are shown for the six time steps in the region near the pilot inlet. These histograms suggest that the vorticity behaviour is similar as it evolves in time. A closer look shows that the non-axial vorticity peak decreases from about 8500 to $6000 \mathrm{~s}^{-1}$ (about 25\%) during the phase of formation of the inner vortex, i.e. from $t_{0}+2 \Delta t$ to $t_{0}+4 \Delta t$, to then re-increase. During the period, the axial vorticity peak moves to the right and towards positive values, reaching a peak of about $2200 \mathrm{~s}^{-1}$ at $t_{0}+2 \Delta t$, immediately before the inner vortex formation. The same qualitative behaviour is observed in terms of mean values, which are also reported in Fig. 7, and by changing the sample domain volume up to $50 \%$ in the pilot region. In the same figure, normalised histograms of mixture fraction suggest the formation of relatively richer mixture at $t_{0}+3 \Delta t$ and $t_{0}+4 \Delta t$. The dashed lines in the same plot showing samples taken for $\widetilde{c}<0.1$ suggest that these richer pockets of mixture are due to the higher temperature gases entrapped in the CRZ. By 


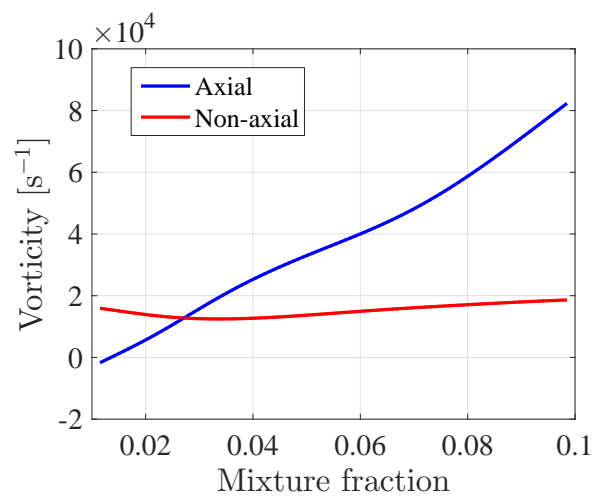

Figure 8: Conditional average of axial and non-axial (magnitude of the two non-axial components) vorticity in the pilot region.

Table 2: Flame position, integrated reaction rate (for main and pilot flames) and injector pressure drop for the six times within the cycle of Fig. 5.

\begin{tabular}{lccc}
\hline Time & $\Delta x / x_{\text {ref }}$ & $Q_{\text {main }} / Q_{0, \text { tot }}$ & $Q_{p} / Q_{0, p}$ \\
\hline$t_{0}=0$ & $1.2-4.2$ & 1.00 & 1.00 \\
$\Delta t$ & $2.0-3.0$ & 1.01 & 0.99 \\
$2 \Delta t$ & $0.8-3.9$ & 1.10 & 1.04 \\
$3 \Delta t$ & $1.7-2.8$ & 1.06 & 0.83 \\
$4 \Delta t$ & $1.8-2.9$ & 1.07 & 1.10 \\
$5 \Delta t$ & $1.4-2.5$ & 1.13 & 0.9 \\
\hline
\end{tabular}

looking at Fig. 8 one can notice that richer pockets of mixture carry higher values of positive axial vorticity. Moreover, the non-axial vorticity is observed to decrease up to about $\widetilde{\xi}=0.03$, which is about the value where the second peak of $\widetilde{\xi}$ is observed on Fig. 7. This behaviour suggests that additional vorticity is brought from relatively richer pockets of mixture in the CRZ and redistributed between axial and non-axial components. The axial vorticity is indeed observed to increase, on average, in the axial direction in the region around the centreline (not shown), suggesting that this vorticity may result from the flame effect. Another possibility is that these relatively richer pockets increase their vorticity as they pass through the shears produced by the CRZ and the IRZ around time $t_{0}+3 \Delta t$, before they move to lower values of $\widetilde{\xi}$ as consequence of the increase in mixing.

Additional insight is provided in Table 2, where pilot flame position (distance from the combustor entrance, $\Delta x / x_{\text {ref }}$ ), and overall reaction rate, $Q=\int_{\mathcal{V}} \overline{\dot{\omega}}_{c} \mathrm{~d} \mathcal{V}$ (normalised by $Q_{0}=Q\left(t=t_{0}\right)$, and where $\mathcal{V}$ is the combustor volume), are shown for the six times of Fig. 5. It is not straightforward to indicate one single value for the pilot flame position as it is extends also in the axial direction 
during the time evolution, so a range of positions is provided instead, which is consistent with the description of flame movement provided earlier. As the flame moves, the heat release rate also oscillates. This oscillation is identified by the variation of $Q_{p}$ and $Q_{\text {main }}$, respectively for pilot and main flames. The integrated reaction rate shows to remain almost constant or slightly increase in both main and pilot flames until $t_{0}+3 \Delta t$, which is when the inner recirculation zone forms inside the pilot jet. At this point $Q$ exhibits a sudden drop, which is relatively small (about 4\%) in the main flame, but relatively high (about $20 \%$ ) in the pilot flame as compared to the value in the previous time step. These variations suggest that the main flame is affected only slightly by the flow variations shown in Fig. 5. Moreover, a similar variation was observed in the peak non-axial vorticity in Fig. 7, suggesting a link among these parameters.

The behaviour observed for Fig. 5 may hide the presence of a precessing motion such as a precessing vortex core (PVC). In this regard additional analyses were performed by looking at the symmetry of cross planes for mean quantities as done for example in [13], at tangential velocity distribution as suggested in [3], and at streamlines evolution in time at a cross section right downstream the injector, $x / x_{\text {ref }}=0.1$, to identify possible presence of radial-axial precessing vortices or other coherent structures. These analyses did not provide any strong evidence of presence of a PVC. However, two vortical structures near the axis were observed to precess around each other in the anticlockwise direction. This is shown in Fig. 9 where streamlines are plotted on top of temperature contours. The two structures carry positive axial vorticity (anticlockwise spin) and correspond to structures $1 \mathrm{a}$ and $1 \mathrm{~b}$ ('fingers') indicated in Fig. 6. They are generated at the interface between the most internal swirled stream from the injector and the next adjacent one, which are counter-rotating. Between these structures there is negative axial vorticity due to the swirling motion, as indicated in Fig. 13 (structure 0n); however this vorticity does not seem to be carried by any definite coherent structure at time $t_{0}$. At time $t_{0}+3 \Delta t$ a $0 \mathrm{n}$ vortex carrying negative axial vorticity is formed and additional vortices ( $3 \mathrm{a}$ and $3 \mathrm{~b}$ in the figure), carrying positive axial vorticity, also appear. The 1a vortex also looks to break down into two smaller vortices (still with positive axial vorticity). The $0 \mathrm{n}$ vortex is associated with the formation of the IRZ as was described for Fig. 5 , and in fact it disappears again at time $t_{0}+4 \Delta t$. The vortices $3 \mathrm{a}$ and $3 \mathrm{~b}$ do not result from the inlet vorticity as can be inferred by comparison with Fig. 6, but rather form from the interaction with the CRZ (vortices $3 \mathrm{a}$ and $3 \mathrm{~b}$ are carrying hot gases, unlike the other structures which are observed to be associated to the cold reactants in Fig. 9) and possibly the IRZ itself. Thus, these two structures increase the overall level of vorticity in the pilot region following the behaviour described for Fig. 7, to then also disappear at time $t_{0}+4 \Delta t$, suggesting a redistribution of vorticity happening around this time. The vortices $1 \mathrm{a}$ and $1 \mathrm{~b}$ complete half revolution around each other at a frequency of about $400 \mathrm{~Hz}$, after which they invert their role. Nevertheless, this movement and the intermittent formation of other vortices at time $t_{0}+3 \Delta t$ do not suggest presence of any lasting off-centered vortex and thus the existence of 

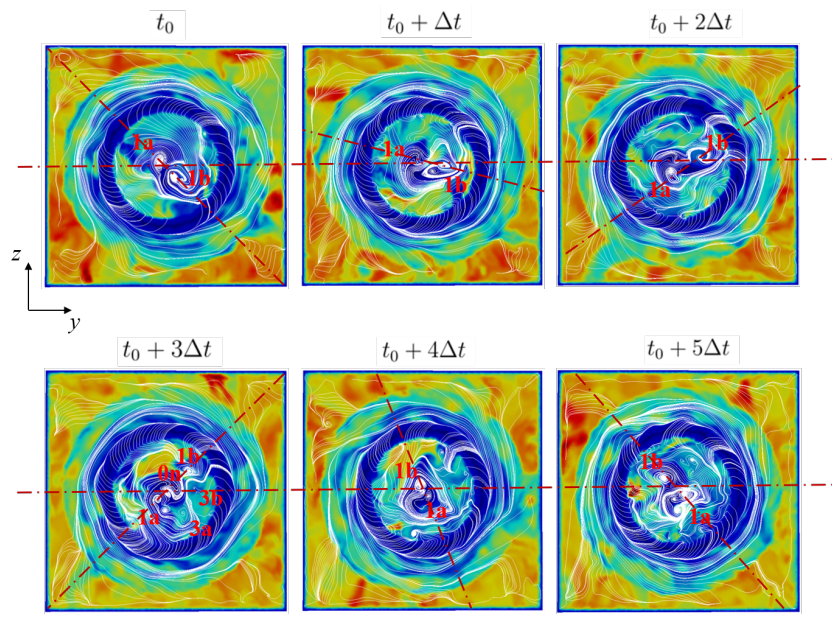

Figure 9: Streamlines are plotted on top of temperature contours for different time steps in the cross section $x / x_{\text {ref }}=0.1$. Blue and red colours identify cold and hot regions respectively. Different vortical structures are marked and labelled. The dashed lines indicate the $y=0$ position and the relative alignment between the structures $1 \mathrm{a}$ and $1 \mathrm{~b}$.

a PVC. It is worth noting that structures 2a and 2b indicated in Fig. 6 would not be seen in Fig. 9 as they carry non-axial vorticity.

By the analyses above two mechanisms are identified, related to each other:

1. A primary mechanism controlled by the vorticity coming from the inlets and modified and redistributed after interaction with the CRZ. Changes in vorticity can result in both a slower mixing, which allows the flame to move upstream driven by richer pockets of mixture; and a less energetic jet that opens allowing the formation of a vortex inside it. This in turn creates a shear layer and a region of small velocities that may allow the flame to stabilise around it;

2. A secondary mechanism is due to the flame itself, since the thermal expansion can produce a decrease in the vorticity around the flame, thus feeding the primary mechanism. More details on this will be given in the next section.

The secondary effect is not observed (or is weak) in Fig. 5 because, as observed earlier, the vortex inside the jet has almost disappeared at the time of maximum flame strength, $t_{0}+4 \Delta t$, and thus the flame never gets too close to the vortex. Moreover, the decrease of vorticity due to the flame dilatation effect must be secondary in this configuration because in the region of formation of the vortex at $t_{0}+3 \Delta t$ no significant change of temperature is observed to justify a change in vorticity, which thus must be changing because of the inlets. 
Let's note that a periodic movement of the the flame and oscillation of heat release were identified for a slightly different condition (slightly lower operating pressure, preheated temperature and air-to-fuel ratio) in [18]. In particular, the work in [18] showed a pulsating motion of the pilot flame at a frequency of about $300 \mathrm{~Hz}$, accompanied by a pulsating transport of liquid fuel and velocity fluctuations. Also, the formation of a temporary inner recirculation zone in front of the pilot injector was observed during the pilot cycle, similarly to what described for Fig. 5. A similar pulsating motion of HR was observed also in the experimental work in [5] at about $400 \mathrm{~Hz}$ for a different combustor, when the Reynolds number was relatively high. This motion was accompanied by formation of ring-vortex structures at the same frequency. Evidence of the mechanism described in the present study was not instead provided during the initial experimental campaign in 2011 for the investigated operating condition [16, 17]. However, the many simultaneous $\mathrm{OH}$ and Kerosene LIF measurements taken during this campaign showed a highly turbulent pilot flow. This can lead to fluctuations of equivalence ratio and formation of rich pockets as also observed in previous works $[59,60,3,12,4]$, which in turn can lead to periodic phenomena or instabilities. Thus, the flow in the experiment may have been prone to the periodic mechanism described numerically in our work.

In order to further explore the two mechanisms described above, the TCI parameters of the combustion model are varied in the next section to obtain changes in vorticity either from the primary or secondary mechanism, and see whether these changes lead to a flame stabilising around the region where the vortex forms, as suggested in this section.

\subsubsection{Flame shape analysis}

Primary and secondary mechanisms controlling the pilot jet angle discussed in the previous section can be directly altered in the LES by changing the TCI parameters. In this way these two effects can be studied separately. In order to affect the primary mechanism, the sub-grid stress model is changed as it has a direct effect on the turbulence, thus on the vorticity, level generated at the inlets which then propagates in the combustion chamber. An additional simulation was thus run using a Vreman model [61] in place of the constant Smagorinsky model and keeping all other parameters identical. It is worth mentioning that the choice of the Vreman model is made with the only intent to alter the vorticity level and distribution and not for a one to one comparison with the Smagorinsky model which would instead require more care to be performed. The secondary mechanism is related to the strength of the pilot flame, which can be increased by artificially decreasing the amount of progress variable SGS variance on the flame area. This was obtained in an additional simulation by setting the reaction term in Eq. (5) to zero and using a linear relaxation model for the SDR to not overbalance the turbulent production. These three different simulations are summarised in Table 3.

Mid-plane mean velocity streamlines for the three cases of Table 3 are shown on top of mean temperature contours in the top row of Fig. 10. For Case A the 
Table 3: The three different simulations for the BOSS rig

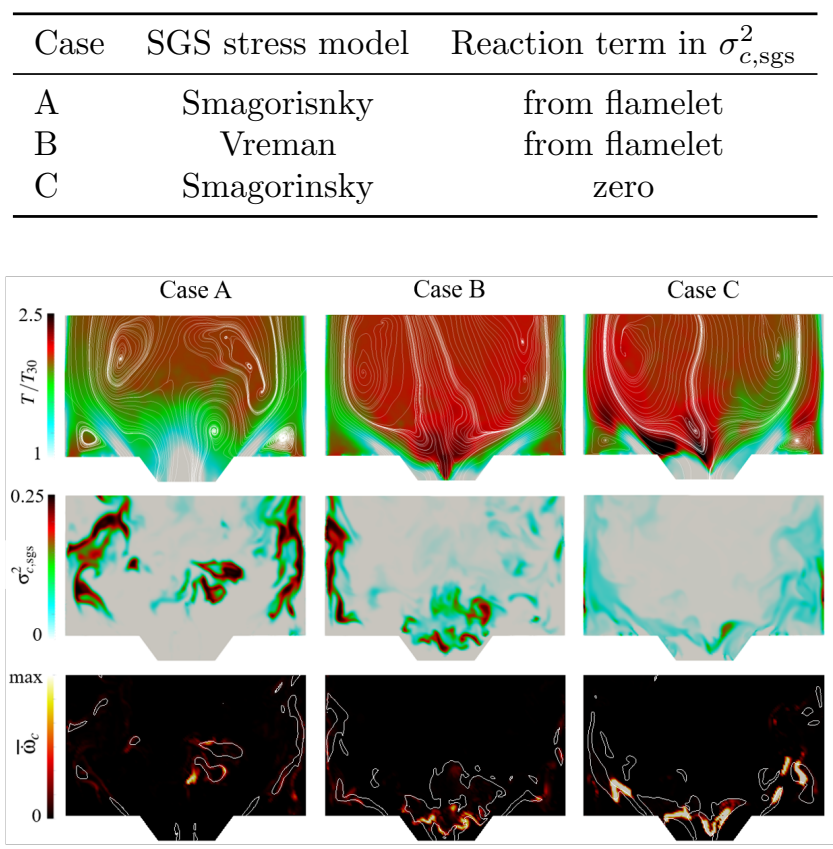

Figure 10: Mid-plane contours of mean (Favre-averaged) temperature (normalised by $T_{30}$ ), and instantaneous progress variable SGS variance, $\sigma_{c, \mathrm{sgs}}^{2}$ and reaction rate, $\overline{\dot{\omega}}_{c}$ from LES are shown for the three cases of Table 3. Mean velocity streamlines and the $\widetilde{\xi}=0.032$ isoline are also shown on top of the temperature and reaction rate contours respectively.

pilot jet is penetrating with two recirculation zones (one annular recirculation from a 3D perspective) around it. The flame in this case has an M-shape as can be inferred by the temperature contour. This shape has moved to a V-flame configuration in cases $\mathrm{B}$ and $\mathrm{C}$ as can be observed in the figure. The mean flow field for these two cases is similar as indicated by the streamlines, with the pilot jet opening early upstream and with a conical angle of about $45^{\circ}$. This diverging jet does not penetrate the combustion chamber in this configuration, and instead two large vortices are observed in front of the injector. It is worth to notice that the flame in this case is still lifted, i.e. it does not flashback, although its position is undesirable being too close to the injector surfaces. Note that a periodic behaviour similar to that observed for Case A is not observed for Cases $\mathrm{B}$ and $\mathrm{C}$. This may be due because the $\mathrm{V}$-flame configuration is more stable due to the very hot region of low velocity and the possible diffusion-like regime, as observed for example in [13].

The physics leading to the V-shape configuration is different between cases $\mathrm{B}$ and $\mathrm{C}$. By comparing the flames between cases A and B at a random instant 


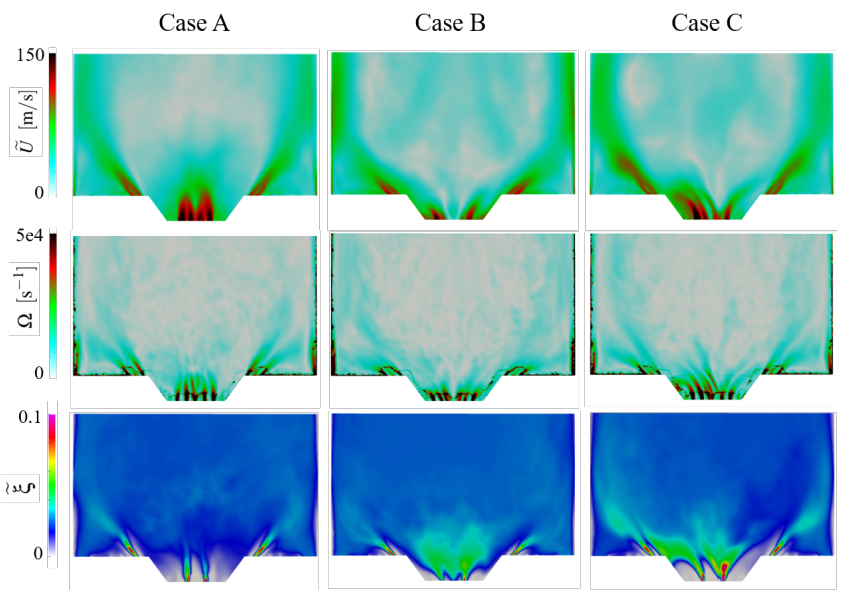

Figure 11: Mid-plane contours of mean (Favre-averaged) velocity, $\widetilde{U}$, vorticity, $\Omega$, and mixture fraction, $\widetilde{\xi}$ from LES are shown for the three cases of Table 3.

of time (see third row of Fig. 10) it is evident that the pilot flame in Case B has moved upstream without changing its strength. This suggests that this movement is caused by the appearance of a lower velocity region due to the formation of a vortex at early stages, as discussed for Fig. 5, and is confirmed by the fact the region of high $\sigma_{c, s g s}^{2}$ values (affecting the flame strength in the flamelet model) has just moved upstream consistently with the movement of the flame (see middle row of Fig. 10), but has not changed significantly in magnitude. For case $\mathrm{C}$ the flame is located at about the same position as Case $\mathrm{B}$, but is much stronger than for Case B as can be observed in the figure. The increased strength is caused by the significantly lower $\sigma_{c, \mathrm{sgs}}^{2}$ artificially imposed, as can be observed in the corresponding contour in Fig. 10. This suggests that in Case $\mathrm{C}$ the flame has started to accelerate upstream causing a deviation of the streamlines and eventually a configuration shift. By a close look at the streamlines for cases $\mathrm{B}$ and $\mathrm{C}$, one can in fact notice that the streamlines in the pilot jet enter the combustion chamber with an angle already in Case B, while they are deviated to form an angle later in Case C, indicating an induced effect.

Additional insights are provided next. It was discussed in section 5.2.1 that the vorticity level accompanying the pilot jet flow right downstream the injector exhibits locally periodic drops (the central 'fingers' are less penetrating), and correspondingly the jet opens allowing the formation of a small vortex as observed for Fig. 5. This mechanism is confirmed by the mean vorticity contours shown in fig. 11 for the three cases of Table 3. In fact, the strong vorticity coming from the pilot jet penetrates, on average, less in Case B in respect to Case A. This leads to a slower mixing rate and thus a richer, near stoichiometric mixture in the immediate downstream of the injector as can be observed in the third row of the same figure. Given that the flame behaves predominantly in a non-premixed mode as observed in section 5.2.1, the flame in case B is 

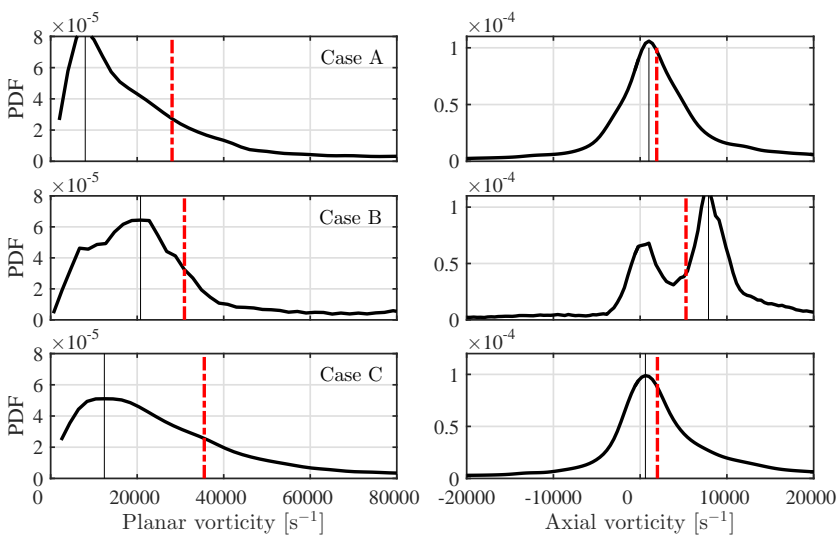

Figure 12: Normalised histograms of axial and planar (magnitude of the two non-axial components) mean vorticity in the pilot region for the three cases of Table 3 . Vertical lines indicate peak (-) and mean (-.-) vorticity.

'entrapped' in this region. It is worth noting that, although the non-premixed mode is dominant, the SGS variance of progress variable (thus the SGS fluctuations of heat release) is strong as was observed in Fig. 10. For Case A the mean vorticity from the pilot jet penetrates more in the combustion chamber and do not show deflection in respect to the centreline. This indicates that the small vortex forming periodically for this configuration (see Fig. 5) does not last enough to impart significant changes to the flow structure, as argued in section 5.2.1. A small jet deviation is observed, however, in the mean velocity contours (top row of Fig. 11), although the mean velocity remains positive at this location.

Some different considerations are needed for Case C. Although the flame position and streamlines are similar to those in Case B as observed earlier, the mean vorticity from the pilot jet as shown in Fig. 11 does not seem to locally drop as it moves into the chamber, but rather to be deflected. However, the non-premixed nature of the pilot flame needs a nearly stoichiometric region to be stabilised. Given that the boundary conditions for cases B and C are identical, it is the flame itself in Case $\mathrm{C}$ to induce the richer mixture region (see bottom row of Fig. 11) in respect to Case A. Note that the flame in Case C is likely to be already at its maximum strength (and thus cannot move further upstream) as it is burning at near stoichiometric conditions (see also mixture fraction iso-lines in Fig. 10) and it would be unphysical to further reduce the progress variable SGS variance since this quantity is already small for this case and a minimum level has to exist due to the effect of the turbulent production (see Eq. (5)).

Further insight is provided by looking at normalised histograms of different vorticity components in the region just downstream the injector marked in Fig. 5. These histograms are shown for the three cases of Table 3 in Fig. 12 for axial and planar (magnitude of the two non-axial components) vorticity. The 


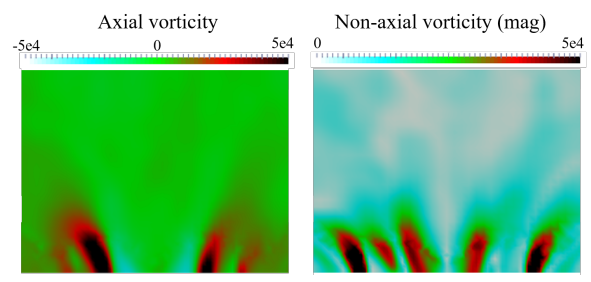

Figure 13: Contours of axial and non-axial (magnitude of the non-axial components) mean vorticity $\left(\right.$ in s $^{-1}$ ) for Case $\mathrm{B}$ of Table 3 , in the zoomed region marked in Fig. 5.

behaviour of mean vorticity observed for Case A is similar to that described for instantaneous vorticity in Fig. 7: there are five vorticity 'fingers' as shown in Fig. 11 of which the internal three are dominated by negative (internal 'finger') and positive (next two external 'fingers') axial vorticity. The peak value is observed to be about $1000 \mathrm{~s}^{-1}$ suggesting a balance slightly in favour of the positive axial vorticity. The two most external 'fingers' are instead dominated by non-axial vorticity as was shown for Fig. 6 and have a peak at about 4000 $\mathrm{s}^{-1}$ as shown by the histogram in Fig. 12. The histograms for Case $\mathrm{C}$ show a behaviour very similar to that of Case A despite the different flame configuration. This support the previous argument that the vorticity in this configuration is deflected and redistributed. In this configuration five vorticity 'fingers' are still observable in Fig. 11 and they are distributed among axial and non-axial vorticity as for Fig. 6. Also, the non-axial distribution is observed to be wider than for Case A, resulting in the mean of the distribution to be higher of about $30 \%$. A similar increase is observed for the peak value. It is worth mentioning that the contours for Case C in Figs. 10 and 11 exhibit a significant asymmetry, suggesting that the simulation time required for this case may be longer than for the other cases, the reason for which will be investigated in a future study. Thus, the data discussed above for Case $\mathrm{C}$ is only qualitative.

A different situation occurs for Case B. First of all, there are six, rather than five, vorticity fingers observable in Fig. 11. Moreover, the two most internal ones are not dominated by axial vorticity anymore, but there is a balance of both negative axial vorticity and non-axial vorticity, which is slightly in favour of the second one. This can be observed in Fig. 13, where contours of these two components are shown. As for Case A, the most external vorticity 'fingers' are dominated by non-axial vorticity while the intermediate ones by positive axial vorticity. The different spacial distribution reflects in the normalised histograms of Fig. 12. The non-axial vorticity distribution as observed for this figure is wider than for case $\mathrm{A}$ and its peak value is more than twice that for the M-flame configuration, although the mean is only observed to increase of about $7 \%$. More interestingly, the axial vorticity presents a marked second peak at about $8000 \mathrm{~s}^{-1}$, i.e. 8 times larger than for Case A. 
The above discussion shows that the vorticity distribution in the pilot region and its evolution are the primary mechanism to determine an M-shape or V-shape configuration. The local distribution of axial vorticity seems to have particular relevance when this quantity does not penetrate far enough into the chamber, near the centreline (vorticity 'fingers' in Figs. 5 and 11). Nevertheless, the histograms in Fig.12 also indicate that this redistribution is accompanied by higher peaks of both axial and non-axial vorticity, probably caused by the interaction with the CRZ as discussed in Section 5.2.1. The secondary mechanism due to the heat release rate contributes instead to a stronger stabilisation of the flame, given that the flame induces the divergence of the streamlines close to the inlet. Nevertheless, it is improbable that the secondary mechanism alone would lead to a change from $\mathrm{M}$-shape to $\mathrm{V}$-shape as long as the richest pockets of mixture are far enough from the inlets. This is because the heat release fluctuation level cannot be controlled in reality as done artificially for the simulation of Case C. However, this simulation suggests the importance of the modelling of the progress variable variance in the context of flamelets, and in particular of the delicate balance between turbulent production, reaction and SDR terms in Eq. (5) which could trigger the secondary mechanism if not evaluated in a satisfactory manner on a physical basis. This has to be kept in mind for modelling perspectives in order to predict the correct configuration in real aero-engines. Also, the relevance of the vorticity level shown in the current analysis gives hints in the way of controlling the flame anchoring position in modern gas turbines, as the vorticity level can be altered at the inlet by changing for example section diameter, surface roughness and swirl number. In

particular the latter can be determinant as it affects the vortex breakdown as identified in [5], where a similar pulsating motion to that investigated in the present work was studied. The same work also suggested an influence of the ORZ. Other controlling parameters were suggested in [18] for the same burner used in the present work, and identified in the injector pressure drop, air-to-fuel ratio and pilot fuel split. Although some data was provided within this work, it is difficult to indicate with precision how much the vorticity should change to trigger a shift in configuration as this requires the study of the evolution during the flame transition, which will be done in a future work. Moreover, the complex interaction between flow-dynamics, spray combustion and geometrical parameters makes it difficult to isolate one single controlling parameter, so the above indications have to be evaluated case by case.

\section{Conclusions}

Large eddy simulations of the reacting flow in a Rolls-Royce rig have been conducted at conditions relevant for aero-engines. The high pressure rig configuration involves preheated swirled air entering the combustion chamber via injectors and kerosene sprayed via atomisers, resulting in a globally lean, partially premixed operating mode. The swirled configuration consists in a central pilot and a surrounding, main jet. The swirled jets contribute to the forma- 
tion of a central vortex core where the burnt gases are entrapped, providing the stabilisation mechanism for the flame. However, the intricate turbulencecombustion interaction can lead to two configurations: i) a penetrating pilot jet with an M-shaped flame, which is also observed in experiments conducted for this configuration; or ii) a diverging pilot jet with a V-shaped flame. The causes leading to the formation of the first or second configuration have been investigated in this work.

A flamelet-based model for LES of partially premixed combustion, employing a revised formulation for the SDR in the progress variable SGS variance equation, has been employed for this analysis. LES results have been firstly compared with experimental data available, and show that the numerical model used is able to satisfactory predict the spray characteristics and the correct flame configuration. A further analysis of these results show a flipping movement of the pilot jet with the periodic formation of a small vortex inside this jet. The numerical analysis of this movement indicates that the pilot jet reaches its maximum divergence of about $45^{\circ}$ when the vorticity level in the pilot region does not penetrate significantly into the chamber near the centreline and redistribute spatially, so to alter the mixing rate and leading to the development of richer pockets of mixture which drive a flame movement upstream. If the vorticity from the inlet is not sufficiently penetrating, the jet divergence can last long enough to cause the pilot flame to move upstream and stabilise on a V-shape. This behaviour, which is accompanied by a redistribution between axial and planar (non-axial) vorticity, has been identified as primary mechanism controlling the shift from M-shape to V-shape and has been verified by conducting a separate simulation where the SGS stress model was changed, resulting in a less penetrating vorticity.

A secondary mechanism has also been identified, which is induced by the flame itself when it gets close to the injectors as consequence of the primary mechanism. The flame can in fact disturb the vorticity isolines, inducing the local formation of richer pockets of mixture upstream, thus contributing to the stabilisation there as a V-shape. This behaviour has been investigated with an additional simulation where the level of SGS variance in the combustion model was artificially decreased by altering the balance between turbulent production, reaction and dissipation rate in order to increase the flame strength.

From a design perspective, the V-shape configuration is undesirable because too close to the injector surfaces. The analysis in this work suggests that this configuration can be avoided by playing with the inlet vorticity so to ensure an homogeneous distribution in the region downstream the injector. This is because the $\mathrm{V}$-shape configuration is unlikely to be caused by the secondary mechanism alone, since the level of SGS fluctuations of heat release cannot be directly controlled in reality as done in the simulation. However an incorrect balance of the source terms in the SGS variance equation of progress variable can trigger this mechanism, which indicates the fundamental role of the above balance at least in the context of flamelet modelling. 


\section{Acknowledgements}

The authors acknowledge funding from the Clean Sky 2 Joint Undertaking under the European Union's Horizon 2020 research and innovation programme (grant agreement No 686332). The authors would like to thank Dr Christoph Hassa for his fruitful comments and valuable suggestions on the manuscript. Dr Andrea Giusti is kindly acknowledged for his contribution to the spray routines implemented in Precise-UNS and for the useful discussions and advice during the review phase.

The LES used for the analysis presented here were performed while IL was at Cambridge University.

\section{References}

[1] Gicquel, L. Y. M., Staffelbach, G., and Poinsot, T., 2012. "Large eddy simulations of gaseous flames in gas turbine combustion chambers". Progress in Energy and Combustion Science, 38(6), pp. 782-817.

[2] Syred, N., and Beér, J. M., 1974. "Combustion in swirling flows: A review". Combust. Flame, 23(2), pp. 143-201.

[3] Syred, N., 2006. "A review of oscillation mechanisms and the role of the precessing vortex core (PVC) in swirl combustion systems". Prog. Energ. Combust. Sci., 32, pp. 93-161.

[4] Steinberg, A. M., Arndt, C. M., and Meier, W., 2013. "Parametric study of vortex structures and their dynamics in swirl-stabilized combustion". Proc. Combust. Int., 34, pp. 3117-3125.

[5] Kraus, C., Harth, S., and Bockhorn, H., 2016. "Experimental investigation of combustion instabilities in lean swirl-stabilized prtially-premixed flames in single- and multiple-burner setup". Spray Combust. Dyn., 8, pp. 4-26.

[6] Rayleigh, J. W. S., 1878. "The explanation of certain acoustic phenomena". Nature, 18, pp. 319-321.

[7] Nicoud, F., and Poinsot, T., 2005. "Thermoacoustic instabilities: should the Rayleigh criterion be extended to include entropy changes". Combust. Flame, 142, pp. 153-159.

[8] Lieuwen, T., and Yang, V., 2005. "Combustion instabilities in gs turbine engines: operational experience, fundamental mechanisms and modeling". In Progress in Astronautics and Aeronautics, Vol. 210, AIAA, American Institute of Aeronautics and Astronautics.

[9] Ducruix, S., Schuller, T., Durox, D., and Candel, S., 2003. "Combustion dynamics and instabilities: Elementary coupling and driving mechnisms". J. Prop. Power, 19, pp. 722-734. 
[10] Driscoll, J. F., and Temme, J., 2011. "Role of swirl in flame stabilization". In 49th AIAA Aerospace Sciences Metting, AIAA, American Institute of Aeronautics and Astronautics.

[11] O'Connor, T., and Lieuwen, T., 2011. "Disturbance field characteristics of a transversely excited burner". Combust. Sci. Technol., 183, pp. 427-443.

[12] Duwig, C., and Fuchs, L., 2007. "Study of flame stabilization in a swirling combustor using a new flamelet formulation". Combust. Sci. Technol., 177, pp. $1485-1510$.

[13] Cheneau, B., Vié, A., and Ducruix, S., 2018. "Numerical study of flame shapes and structures in a two-stage two-injection aeronautical burner with variable fuel staging using Eulerian large eddy simulations". J. Eng. Gas Turbines Power.

[14] Sommerer, Y. nd Galley, D., Poinsot, T., Ducruix, S., Lacas, F., and Veynante, D., 2004. "Large eddy simulation and experimental study of flashback and blow-off in a lean partially premixed swirled burner". J. Turbul., 5, pp. 1-21.

[15] Kohse-Höinghaus, K., Barlow, R. S., Aldén, M., and Wolfrum, J., 2005. "Combustion at the focus: Laser diagnostics and control". Proceedings of the Combustion Institute, 30(1), pp. 89-123.

[16] Schneider, D., Meier, U., Quade, W., Koopman, J., Aumeier, T., Langfeld, A., Behrendt, T., Hassa, C., and Rackwitz, L., 2010. "A new test rig for laser optical investigations of lean jet burners". Proc. 27th Int. Congress Aeronaut. Sci.

[17] Freitag, S., Behrendt, T., Heinze, J., Lange, L., Meier, U., Rackwitz, L., and Hassa, C., 2011. "Study of an airblast atomizer spray in a lean burn aero-engine model combustor at engine conditions". Proc. ILASSEurope.

[18] Meier, U., Lange, L., Heinze, J., Hassa, C., Sadig, S., and Luff, D., 2014. "Optical methods for studies of self-excited oscillations and the effect of dampers in a high pressure single sector combustor". Proc. ASME Turbo Expo (GT2014-25873).

[19] Pope, S. B., 2000. "Large-eddy simulation". In Turbulent Flows. Cambridge University Press, Cambridge, ch. 13.

[20] Poinsot, T. J., and Veynante, D., 2005. Theoretical and numerical combustion, second ed. Edwards.

[21] Langella, I., and Swaminathan, N., 2015. "Unstrained and strained flamelets for LES of premixed combustion". Combustion Theory and Modelling, 20(3), pp. 410-440.

doi:10.1080/13647830.2016.1140230. 
[22] Langella, I., Swaminathan, N., and Pitz, R. W., 2016. "Application of unstrained flamelet SGS closure for multi-regime premixed combustion". Combustion and Flame, 173, Nov., pp. 161-178.

[23] Langella, I., Swaminathan, N., Williams, F. A., and Furukawa, J., 2016. "Large-eddy simulation of premixed combustion in the corrugated-flamelet regime". Combustion Science and Technology, 188(9), pp. 1565-1591.

[24] Chen, Z., Ruan, S., and Swaminathan, N., 2017. "Large eddy simulation of flame edge evolution in a spark-ignited methane-air jet". Proc. Combust. Inst., 36, pp. 1645-1652.

[25] Langella, I., Chen, Z. X., Swaminathan, N., and Sadasivuni, S. K., 2018. "Large-eddy simulation of reacting flows in industrial gas turbine combustor". J. Propul. Power, 34, pp. 1269-1284.

[26] Dunn, M. J., Masri, A. R., Bilger, R. W., Barlow, R. S., and Wang, G. H., 2009. "The compositional structure of highly turbulent piloted premixed flames issuing into a hot coflow". Proceedings of the Combustion Institute, 32(2), pp. 1779-1786.

[27] Dunn, M. J., Masri, A. R., Bilger, R. W., and Barlow, R. S., 2010. "Finite rate chemistry effects in highly sheared turbulent premixed flames". Flow Turbulence Combust., 85, pp. 621-648.

[28] Poludnenko, A. Y., and Oran, E. S., 2010. "The interaction of high-speed turbulence with flames: Global properties and internal flame structure". Combust. Flame, 157, pp. 995-1011.

[29] Temme, J. E. nd Wabel, T. M., Skiba, A. W., and Driscoll, J. F., 2015. "Measurements of premixed turbulent combustion regimes of high Reynolds number flames". In 53rd AIAA Aerospace Sciences Metting, AIAA, American Institute of Aeronautics and Astronautics.

[30] Poinsot, T., Veynante, D., and Candel, S., 1991. "Quenching processes and premixed turbulent combustion diagrams". J. Fluid Mech., 228, pp. 561606.

[31] Roberts, W. L., Driscoll, J. F., Drake, M. C., and Goss, L. P., 1993. "Images of the quenching of a flame by a vortex - to quantify regimes of turbulent combustion". Combustion and Flame, 94(1-2), pp. 58-69.

[32] Duwig, C., 2007. "Study of a filtered flame formulation for large eddy simulation of premixed turbulent flames". Flow Turb. Combust., 79(4), pp. 433-454.

[33] Giusti, A., Mastorakos, E., Hassa, C., Heinze, J., Magens, E., and Zedda, M., 2018. "Investigation of flame structure and soot formation in a single sector model combustor using experiments and numerical simulations based on the large eddy simulation/conditional moment closure approach". J. Eng. Gas Turbines Power, 140, pp. 061506-061506-9. 
[34] Heinze, J., Meier, U., Behrendt, T., Willert, C., Geigle, K.-P., Lammel, O., and Lückerath, R., 2011. "PLIF thermometry based on measurements of absolute concentrations of the OH radical". Z. Chem. Phys., 225, pp. 13151341.

[35] Lange, L., Heinze, J., Scholl, M., Willert, C., and Behrendt, T., 2012. "Combination of planar laser optical measurement techniques for the investigation of pre-mixed lean combustion". In 16th International Symposium on Applications of Laser Techniques to Fluid Mechanics.

[36] Swaminathan, N., and Bray, K. N. C., 2011. "Fundamentals and challenges". In Turbulent Premixed Flames, N. Swaminathan and K. N. C. Bray, eds. Cambridge University Press, Cambridge, UK, ch. 1, pp. 1-40.

[37] Knudsen, E., and Pitsch, H., 2012. "Capabilities and limitations of multiregime flamelet combustion models". Combustion and Flame, 159(1), pp. $242-264$.

[38] Srinivasan, S., and Menon, S., 2014. "Linear eddy mixing model studies of high Karlovitz number turbulent premixed flames". Flow Turbulence and Combustion, 93(2), pp. 189-219.

[39] Bilger, R. W., Stoårner, S. H., and Kee, R. J., 1990. "On reduced mechanism for methane-air combustion in nonpremixed flames". Combust. Flame, 80, pp. $135-149$.

[40] Demoulin, F. X., and Borghi, R., 2002. "Modeling of turbulent spray combustion with application to diesel like experiment". Combust. Flame, 129, pp. 281-293.

[41] Fiorina, B., Baron, R., Gicquel, O., Thevenin, D., Carpentier, S., and Darabiha, N., 2003. "Modelling non-adiabatic partially premixed flames using flame-prolongation of ILDM". Combustion Theory and Modelling, 7(3), pp. 449-470.

[42] Davis, P. J., 1970. "Gamma functions and related functions". In Handbook of mathematical functions, M. Abramowitz and I. A. Stegun, eds. Dover Publications Inc., New York.

[43] Pera, C., Réveillon, J., Vervisch, L., and Domingo, P., 2006. "Modeling subgrid scale mixture fraction variance in les of evaporating spray". Combust. Flame, 146(4), pp. $635-648$.

[44] Pitsch, H., 2006. "Large-eddy simulation of turbulent combustion". Annual Reviews of Fluid Mechanics, 38, Jan., pp. 453-482.

[45] Giusti, A., Kotzagianni, M., and Mastorakos, E., 2016. "LES/CMC simulations of swirl-stabilised ethanol spray flames approaching blow-off". Flow Turb. Combust., 97, pp. 1165-1184. 
[46] Chrigui, M., Gounder, J., Sadiki, A., Masri, A. R., and Janicka, J., 2012. "Partially premixed reacting acetone spray using les and fgm tabulated chemistry". Combust. Flame, 159(8), pp. 2718 - 2741.

[47] Dunstan, T. D., Minamoto, Y., Chakraborty, N., and Swaminathan, N., 2013. "Scalar dissipation rate modelling for Large Eddy Simulation of turbulent premixed flames". Proceedings of the Combustion Institute, 34(1), pp. 1193-1201.

[48] Langella, I., Swaminathan, N., Gao, Y., and Chakraborty, N., 2016. "LES of premixed combustion: Sensitivity to SGS velocity modelling". Combustion Science and Technology, published online 21 Jun.

[49] Langella, I., Doan, N. A. K., and Swaminathan, N.and Pope, S. B., 2018. "Study of subgrid-scale velocity models for reacting and nonreacting flows". Phys. Rev. Fluids, 3, p. 054602.

[50] Langella, I., Swaminathan, N., Gao, Y., and Chakraborty, N., 2015. "Assessment of dynamic closure for premixed combustion LES". Combustion Theory and Modelling, 19(5), pp. 628-656.

[51] Anand, M. S., Eggels, R., Staufer, M., Zedda, M., and Zhu, J., 2013. "An advanced unstructured-grid finitevolume design system for gas turbine combustion analysis". Proc. ASME Gas Turbine India (GTINDIA20133537).

[52] Doormaal, J. P. V., and Raithby, G. D., 1984. "Enhancements of the simple method for predicting incompressible fluid flows". Numerical Heat Transfer, $\mathbf{7}(2)$, pp. 147-163.

[53] Dagaut, P., and Cathonnet, M., 2006. "The ignition, oxidation, and combustion of kerosene: A review of experimental and kinetic modeling". Prog. Energy Combust. Sci., 32, pp. 48-92.

[54] Lilly, D. K., 1967. "The representation of small-scale turbulence in numerical simulation experiments". In Proceedings of the IBM Scientific Computing Symposium on Environmental Sciences, H. H. Goldstine, ed., no. 3201951. IBM, pp. 195-210.

[55] Gepperth, S., Koch, R., and Bauer, H.-J., 2013. "Analysis and comparison of primary droplet characteristics in the near field of a prefilming airblast atomizer". Proc. ASME Turbo Expo(GT2013-94033).

[56] Schmehl, R., Maier, G., and Wittig, S., 2000. "CFD analysis of fuel atomization, secondary droplet breakup and spray dispersion in the premix duct of a LPP combustor". Proc. of the ICLASS.

[57] Miller, R., H., and K., Bellan, J., 1998. "Evaluation of equilibrium and non-equilibrium evaporation models for many-droplet gas-liquid flow simulations". Int. J. Multiphase Flow, 24, pp. 1025-1055. 
[58] Batchelor, G. K., 1967. Introduction to Fluid Mechanics. Cambridge University Press.

[59] Schildmacher, K.-U., Koch, R., Krebs, W., Hoffmann, S., and Wittig, S., 2000. "Experimental investigations of the temporal air-fuel mixing fluctuations and cold flow instabilities of a premixing gas turbine burner". ASME 200-GT-0084.

[60] Fritz, J., Kr oner, M., and Settelmayer, T., 2001. "Flashback in a swirl burner with cylindrical premixing zone". In International Gas Turbine and Aeroengine Congress and Exhibition, ASME, American Society of Science and Engineering.

[61] Vreman, A. W., 2004. "An eddy-viscosity subgrid-scale model for turbulent shear flow: Algebraic theory and applications". Phys. Fluids, 16, pp. 36703681 . 